\title{
Some New Characterizations Of Parallel Translation Surface According To Bishop Frame With Timelike $M_{1}$ In Minkowski 3-Space
}

\author{
Gülden Altay Suroğlu ${ }^{1}$ \\ ${ }^{1}$ Department of Mathematics, University of Firat, Elazı̆̆, Turkey
}

Abstract. In this paper we consider parallel translation surfaces, which are generated by spacelike curves, according to Bishop frame with timelike $M_{1}$ in Minkowski 3- space. Then, we obtain some characterizations of these surface.

\section{Introduction}

Creation of paralel surfaces is useful in design and manufacture. A surface $M^{r}$ is parallel to $\mathrm{M}$ if points of $M^{r}$ are at a constant distance along the normal from the surface M. So, there are infinite numbers of parallel surfaces, [8].

Kızıltuğ and Yaylı studied timelike curves on timelike parallel surfaces in Minkowski 3 -spaces $E_{1}{ }^{3}$. Then, they gave some characterization for its image curve in, [3].

Unluturk and Ekici obtain paralel surfaces of timelike ruled surfaces which are developable are timelike ruled Weingarten surfaces. Then, some properties of that kind paralel surfaces are obtained in Minkowski 3-space in [7].

Safiulina gave a complete classification of the existence and geometry of such twodimensional Riemannian submanifolds (surfaces). Moreover, it is shown that in $E_{s}^{n}$ with $\mathrm{s}>0$ do exist not totally geodesic minimal semiparallel space-like surfaces, [6].

In [1], Calvaruso and Van der Veken completely classified surfaces with parallel second fundamental form in all non-symmetric homogeneous Lorentzian three manifolds. In [2], Fukui and Hasegawa investigate singularities of all parallel surfaces to a given regular surface.

\section{Fundamental Properties of the Method}

Let $\alpha: I \rightarrow E_{1}{ }^{3}$ be a non geodesic spacelike curve on the $E_{1}{ }^{3}$ parameterized by arc length. Let $\{\mathrm{T}, \mathrm{N}, \mathrm{B}\}$ be the Frenet frame. Then, for spacelike curves we have the following Frenet formulas:

Corresponding Author : guldenaltay23@hotmail.com 


$$
\frac{d}{d s}\left[\begin{array}{l}
\mathbf{T}(s) \\
\mathbf{N}(s) \\
\mathbf{B}(s)
\end{array}\right]=\left[\begin{array}{ccc}
0 & \kappa(s) & 0 \\
-\varepsilon \kappa(s) & 0 & \tau(s) \\
0 & \tau(s) & 0
\end{array}\right]\left[\begin{array}{c}
\mathbf{T}(s) \\
\mathbf{N}(s) \\
\mathbf{B}(s)
\end{array}\right],
$$

where $\kappa \neq 0$ and $\tau \neq 0$ and

$$
\mathrm{g}(\mathrm{T}, \mathrm{T})=1, \mathrm{~g}(\mathrm{~N}, \mathrm{~N})=\varepsilon= \pm 1, \mathrm{~g}(\mathrm{~B}, \mathrm{~B})=1 .
$$

The Bishop frame is an option when the spacelike curve has a vanishing second derivative. The Bishop frame is

$$
\frac{d}{d s}\left[\begin{array}{c}
\mathbf{T}(s) \\
\mathbf{M}_{1}(s) \\
\mathbf{M}_{2}(s)
\end{array}\right]=\left[\begin{array}{ccc}
0 & k_{1}(s) & -k_{2}(s) \\
k_{1}(s) & 0 & 0 \\
k_{2}(s) & 0 & 0
\end{array}\right]\left[\begin{array}{c}
\mathbf{T}(s) \\
\mathbf{M}_{1}(s) \\
\mathbf{M}_{2}(s)
\end{array}\right],
$$

where

$$
\mathrm{g}(\mathrm{T}, \mathrm{T})=-\mathrm{g}\left(\mathrm{M}_{1}, \mathrm{M}_{1}\right)=\mathrm{g}\left(\mathrm{M}_{2}, \mathrm{M}_{2}\right)=1 .
$$

For timelike curves, we have the following Frenet formulas:

$$
\frac{d}{d s}\left[\begin{array}{c}
\mathbf{T}(s) \\
\mathbf{N}(s) \\
\mathbf{B}(s)
\end{array}\right]=\left[\begin{array}{ccc}
0 & \kappa(s) & 0 \\
\kappa(s) & 0 & \tau(s) \\
0 & -\tau(s) & 0
\end{array}\right]\left[\begin{array}{c}
\mathbf{T}(s) \\
\mathbf{N}(s) \\
\mathbf{B}(s)
\end{array}\right]
$$

Then, the Bishop frame is expressed as

$$
\frac{d}{d s}\left[\begin{array}{c}
\mathbf{T}(s) \\
\mathbf{M}_{1}(s) \\
\mathbf{M}_{2}(s)
\end{array}\right]=\left[\begin{array}{ccc}
0 & k_{1}(s) & k_{2}(s) \\
k_{1}(s) & 0 & 0 \\
k_{2}(s) & 0 & 0
\end{array}\right]\left[\begin{array}{c}
\mathbf{T}(s) \\
\mathbf{M}_{1}(s) \\
\mathbf{M}_{2}(s)
\end{array}\right]
$$

Here, the set $\left\{\mathrm{T}, \mathrm{M}_{1}, \mathrm{M}_{2}\right\}$ is called Bishop trihedra, $\mathrm{k}_{1}$ and $\mathrm{k}_{2}$ as Bishop curvatures, $\tau(\mathrm{s})=\theta^{\prime}(\mathrm{s})$ and $\kappa(\mathrm{s})=\sqrt{ }\left(\left|\mathrm{k}_{2}{ }^{2}-\mathrm{k}_{1}{ }^{2}\right|\right)$.

\section{Parallel Translation Surfaces in $E_{1}^{3}$}

Let $\alpha(\mathrm{u})$ and $\beta(\mathrm{v})$ be spacelike curves and $\theta(\mathrm{u}, \mathrm{v})$ be a translation surface which is parametrized as

$$
\theta(\mathrm{u}, \mathrm{v})=\alpha(\mathrm{u})+\beta(\mathrm{v}) .
$$

Then, parallel surfaces of the translation surface is

$$
\phi(\mathrm{u}, \mathrm{v})=\theta(\mathrm{u}, \mathrm{v})+\mathrm{aU}(\mathrm{u}, \mathrm{v})
$$

where a is a constant and $\mathrm{U}(\mathrm{u}, \mathrm{v})$ is unit normal vector field of the translation surface $\theta(\mathrm{u}, \mathrm{v})$. So, it parameterized as

$$
\phi(u, v)=\alpha(u)+\beta(v)+\frac{a}{\sin \alpha}\left(T_{\alpha} \times T_{\beta}\right)
$$

where $\alpha$ is the constant angle between unit tangent vector field of curves $T_{\alpha}$ and $T_{\beta}$.

Theorem 1. Let $\alpha(\mathrm{u})$ and $\beta(\mathrm{v})$ be spacelike curves where $M_{1}^{\alpha}$ and $M_{1}^{\beta}$ are timelike 
vector fields in Minkowski three space. Then if $\phi(\mathrm{u}, \mathrm{v})$ is minimal,

$$
\begin{aligned}
& E\left(-a^{2}\left(k_{1}^{\beta 2}-k_{2}^{\beta 2}\right)^{2}+k_{1}^{\beta}<M_{1}^{\beta}, U>-k_{2}^{\beta}<M_{2}^{\beta}, U>+\frac{a k_{1}^{\beta} 1}{\sin \alpha}<W, U>-\frac{a k_{2}^{\beta} 1}{\sin \alpha}<X, U>\right) \\
& -2 F\left(\frac{a k_{1}^{\beta}}{\sin \alpha}\left(k_{1}^{\beta}<Q, U>-k_{2}^{\beta}<P, U>\right)+\frac{a k_{2}^{\alpha}}{\sin \alpha}\left(k_{1}^{\beta}<R, U>-k_{2}^{\beta}<S, U>\right)\right. \\
& +G\left(-a^{2}\left(k_{1}^{\alpha 2}-k_{2}^{\alpha 2}\right)^{2}+k_{1}^{\alpha}<M_{1}^{\alpha}, U>-k_{2}^{\alpha}<M_{2}^{\alpha}, U>\right. \\
& \left.+\frac{a k_{1}^{\alpha} 1}{\sin \alpha}<W, U>-\frac{a k_{2}^{\alpha}}{\sin \alpha}<X, U>\right)=0 .
\end{aligned}
$$

Proof. If we take derivatives of the parallel surface of the translation surface according to $\mathrm{u}$ and $\mathrm{v}$, then from bishop equations, we have

$$
\phi_{u}(u, v)=T_{\alpha}+\frac{a k_{1}^{\alpha}}{\sin \alpha}\left(M_{1}^{\alpha} \times T_{\beta}\right)-\frac{a k_{2}^{\alpha}}{\sin \alpha}\left(M_{2}^{\alpha} \times T_{\beta}\right) \text {. }
$$

$$
\phi_{v}(u, v)=T_{\beta}+\frac{a k_{1}^{\beta}}{\sin \alpha}\left(T_{\alpha} \times M_{1}^{\beta}\right)-\frac{a k_{2}^{\beta}}{\sin \alpha}\left(T_{\alpha} \times M_{2}^{\beta}\right)
$$

Similarly,

The compenents of the first fundamental form of $\phi(\mathrm{u}, \mathrm{v})$ are

$$
\begin{aligned}
& E=1-a k_{1}^{\alpha}<U, M_{1}^{\alpha}>+a k_{2}^{\alpha}<U, M_{2}^{\alpha}>+\frac{1}{\sin ^{2} \alpha}\left(a k_{1}^{\alpha 2}\left(\left\|M_{1}^{\alpha}\right\|^{2}-<M_{1}^{\alpha}, T_{\beta}>\right)\right. \\
& \left.+a k_{2}^{\alpha 2}\left(\left\|M_{2}^{\alpha}\right\|^{2}-<M_{2}^{\alpha}, T_{\beta}>\right)\right), \\
& F=\cos \alpha+\frac{a^{2} k_{1}^{\alpha} k_{1}^{\beta}}{\sin ^{2} \alpha}\left(<M_{1}^{\alpha}, T_{\alpha}><M_{1}^{\beta}, T_{\beta}>-\sin \alpha<M_{1}^{\alpha}, M_{1}^{\beta}>\right) \\
& -\frac{a^{2} k_{1}^{\alpha} k_{2}^{\beta}}{\sin ^{2} \alpha}\left(<M_{1}^{\alpha}, T_{\alpha}><M_{2}^{\beta}, T_{\beta}>-\sin \alpha<M_{1}^{\alpha}, M_{2}^{\beta}>\right) \\
& -\frac{a^{2} k_{2}^{\alpha} k_{1}^{\beta}}{\sin ^{2} \alpha}\left(<M_{2}^{\alpha}, T_{\alpha}><M_{1}^{\beta}, T_{\beta}>-\sin \alpha<M_{2}^{\alpha}, M_{1}^{\beta}>\right) \\
& -\frac{a^{2} k_{2}^{\alpha} k_{2}^{\beta}}{\sin ^{2} \alpha}\left(<M_{2}^{\alpha}, T_{\alpha}><M_{2}^{\beta}, T_{\beta}>-\sin \alpha<M_{2}^{\alpha}, M_{2}^{\beta}>\right) \\
& G=1-a k_{1}^{\beta}<U, M_{1}^{\beta}>+a k_{2}^{\beta}<U, M_{2}^{\beta}>+\frac{1}{\sin ^{2} \alpha}\left(a k_{1}^{\beta 2}\left(\left\|M_{1}^{\beta}\right\|^{2}-<M_{1}^{\beta}, T_{\alpha}>\right)\right. \\
& \left.+a k_{2}^{\beta 2}\left(\left\|M_{2}^{\beta}\right\|^{2}-<M_{2}^{\beta}, T_{\alpha}>\right)\right) .
\end{aligned}
$$

The unit vector field of the parallel translation surface is

$$
U=\frac{1}{\sqrt{E G} \sin \gamma}\left(\phi_{u} \times \phi_{v}\right)
$$

where $\gamma$ is the angle between vector fields $\phi_{u}$ and $\phi_{v}$.

Then, components of the second fundamental form of the $\phi(u, v)$ are 


$$
\begin{aligned}
& h_{11}=-a^{2}\left(k_{1}^{\alpha 2}-k_{2}^{\alpha 2}\right)^{2}+k_{1}^{\alpha}<U, M_{1}^{\alpha}>-k_{2}^{\alpha}<U, M_{2}^{\alpha}> \\
& +\frac{a k_{1}^{\alpha}{ }^{\prime}}{\sin \alpha}<U, W>-\frac{a k_{2}^{\alpha}{ }^{\prime}}{\sin \alpha}<U, X>, \\
& h_{12}=\frac{a k_{1}^{\alpha}}{\sin \alpha}\left(k_{1}^{\beta}<Q, U>-k_{2}^{\beta}<P, U>\right)+\frac{a k_{2}^{\alpha}}{\sin \alpha}\left(k_{1}^{\beta}<R, U>-k_{2}^{\beta}<S, U>\right), \\
& h_{22}=-a^{2}\left(k_{1}^{\beta 2}-k_{2}^{\beta 2}\right)^{2}+k_{1}^{\beta}<U, M_{1}^{\beta}>-k_{2}^{\beta}<U, M_{2}^{\beta}> \\
& +\frac{a k_{1}^{\beta}}{\sin \alpha}<U, W>-\frac{a k_{2}^{\beta}}{\sin \alpha}<U, X>,
\end{aligned}
$$

Where

$$
\begin{aligned}
& M_{1}^{\alpha} \times T_{\beta}=W, M_{2}^{\alpha} \times T_{\beta}=X, T_{\alpha} \times M_{1}^{\beta}=Y, T_{\alpha} \times M_{2}^{\beta}=Z, M_{1}^{\alpha} \times M_{1}^{\beta}=Q, \\
& M_{1}^{\alpha} \times M_{2}^{\beta}=P, M_{2}^{\alpha} \times M_{1}^{\beta}=R, M_{2}^{\alpha} \times M_{2}^{\beta}=S .
\end{aligned}
$$

Then the proof is complete.

Corollary 1 . Let $\alpha(\mathrm{u})$ be timelike and $\beta(\mathrm{v})$ be spacelike curves where $M_{1}^{\beta}$ is timelike vector field. Then if parallel surfaces of the translation surface $\tilde{\phi}(u, v)$ is maximal,

$$
\begin{aligned}
& \tilde{E}\left(a^{2}\left(k_{1}^{\beta 2}-k_{2}^{\beta 2}\right)^{2}+k_{1}^{\beta}<M_{1}^{\beta}, \tilde{U}>-k_{2}^{\beta}<M_{2}^{\beta}, \tilde{U}>+\frac{a k_{1}^{\beta \prime}}{\cosh \alpha}<W, \tilde{U}>-\frac{a k_{2}^{\beta \prime}}{\cosh \alpha}<X, \tilde{U}>\right) \\
& -2 \tilde{F}\left(\frac{a k_{1}^{\alpha}}{\sin \alpha}\left(k_{1}^{\beta}<Q, \tilde{U}>-k_{2}^{\beta}<P, \tilde{U}>\right)+\frac{a k_{2}^{\alpha}}{\cosh \alpha}\left(k_{1}^{\beta}<R, \tilde{U}>-k_{2}^{\beta}<S, \tilde{U}>\right)\right. \\
& +\tilde{G}\left(a^{2}\left(k_{1}^{\alpha 2}-k_{2}^{\alpha 2}\right)^{2}+k_{1}^{\alpha}<M_{1}^{\alpha}, \tilde{U}>-k_{2}^{\alpha}<M_{2}^{\alpha}, \tilde{U}>\right. \\
& \left.+\frac{a k_{1}^{\alpha}{ }^{\prime}}{\cosh \alpha}<W, \tilde{U}>-\frac{a k_{2}^{\alpha}{ }^{\prime}}{\cosh \alpha}<X, U,>\right)=0
\end{aligned}
$$

where $\tilde{E}, \tilde{F}$ and $\tilde{G}$ are components of the first fundamnetal form.

\section{References}

1. Giovanni Calvaruso and Joeri Van der Veken, Parallel Surfaces in Three Dimensional Lie Groups, Taiwanese Journal of Mathematics, 14, 223-250 (2010)

2. Toshizumi Fukui, Masaru Hasegawa, Singularities of Parallel Surfaces, Tohoku Mathematical Journal, 64, 387-408 (2012)

3. Sezai Kızıltuğ, Yusuf Yayl1, Timelike Curves on Timelike Parallel Surfaces in Minkowski 3-space $\mathrm{E}_{1}{ }^{3}$, Mathematica Aeterna, 2, 689 - 700 (2012)

4. Talat Körpınar, Essin Turhan, Spacelike Biharmonic New Type B-Slant Helices According to Bishop Frame in the Lorentzian Heisenberg Group $\mathrm{H}^{3}$, General Mathematics Notes, 10,36-42 (2012)

5. Talat Körpınar, Essin Turhan, Parallel Surfaces to Normal Ruled Surfaces of General Helices in the Sol Space Sol ${ }^{3}$, Boletim Sociedade Paranaense de. Matematica, 31, 245-253 (2013) 
6. Elena Safiulina, Parallel and Semi- parallel Space-like Surfaces in PseudoEuclidean Spaces,Proc.Estonian Acad. Sci. Phys.Math., 50, 16-33, (2001)

7. Yasin Unluturk, Cumali Ekici, On Parallel Surfaces of Timelike Ruled Weingarten Surfaces, Balkan Journal of Mathematics, 1, 72-91 (2003)

8. Dae Woon Yoon, Some properties of parallel surfaces in Euclidean 3-spaces, Honam Mathematical Journal, 30, 637-644 (2008)

9. Süha Yilmaz, Bishop spherical images of a spacelike curve in Minkowski 3space, International Journal of the Physical Sciences, 5, 898-905 (2010) 